\title{
Parallelised photoacoustic signal acquisition using a Fabry-Perot sensor and a camera-based interrogation scheme
}

T. Saeb Gilani, C. Villringer, E. Zhang, H. Gundlach, J. Buchmann, et al.

T. Saeb Gilani, C. Villringer, E. Zhang, H. Gundlach, J. Buchmann, S. Schrader, J. Laufer, "Parallelised photoacoustic signal acquisition using a Fabry-Perot sensor and a camera-based interrogation scheme," Proc. SPIE 10494, Photons Plus Ultrasound: Imaging and Sensing 2018, 1049431 (20 February 2018); doi: 10.1117/12.2290223 


\title{
Parallelised photoacoustic signal acquisition using a Fabry-Perot sensor and a camera-based interrogation scheme
}

\author{
T. Saeb Gilani*a, C. Villringer ${ }^{\mathrm{b}, \mathrm{c}}$, E. Zhang ${ }^{\mathrm{d}}$, H. Gundlach ${ }^{\mathrm{a}}$, J. Buchmann ${ }^{\mathrm{a}}$, S. Schrader ${ }^{\mathrm{b}}$, J. Laufer ${ }^{\mathrm{e}}$ \\ ${ }^{a}$ Institute of Optics and Atomic Physics, Technical University of Berlin, Germany \\ ${ }^{b}$ University of Applied Science Wildau, Germany \\ ${ }^{c}$ Department of Industrial Engineering, University of Rome, Italy \\ ${ }^{d}$ Department of Medical Physics and Biomedical Engineering, University College London, UK \\ eInstitute of Physics, Martin-Luther University Halle-Wittenberg, Germany
}

\begin{abstract}
Tomographic photoacoustic (PA) images acquired using a Fabry-Perot (FP) based scanner offer high resolution and image fidelity but can result in long acquisition times due to the need for raster scanning. To reduce the acquisition times, a parallelised camera-based PA signal detection scheme is developed. The scheme is based on using a sCMOScamera and FPI sensors with high homogeneity of optical thickness. PA signals were acquired using the camera-based setup and the signal to noise ratio (SNR) was measured. A comparison of the SNR of PA signal detected using 1) a photodiode in a conventional raster scanning detection scheme and 2) a sCMOS camera in parallelised detection scheme is made. The results show that the parallelised interrogation scheme has the potential to provide high speed PA imaging.
\end{abstract}

Keywords: Photoacoustic imaging, Fabry-Perot polymer film sensor, Parallelised signal acquisition

\section{INTRODUCTION}

PA imaging systems based on Fabry-Perot interferometer (FPI) sensors provide high fidelity and high resolution images. FPI sensors exhibit high acoustic sensitivity, small active element sizes and broadband frequency response which allows non-invasive high resolution biomedical imaging to cm-depths [1]-[5].

Current FPI interrogation schemes are based on raster scanning of a focused interrogation laser beam [1] across the FPI sensor. At each scan point, a PA wave is generated in the adjacent target by the absorption of an excitation pulse. The propagation of ultrasonic wave modulates the optical thickness of the FPI sensor, which in turn results in a modulation of the optical power reflected by FPI sensor. PA signals are acquired by recording the time-course of the optical power using a photodiode. Since the signal generation has to be repeated at each scan point, this method typically results in long image acquisition times of several minutes. To overcome this limitation, parallelization approaches are explored, such as the use of multiple interrogation beams [6]. In this study, a camera-based parallelised PA signal acquisition scheme is developed, in which the FPI sensor is illuminated by the collimated beam of the interrogation laser and the acoustically induced modulation of the optical phase, and hence the reflected optical power, is mapped using a sCMOS camera at increasing time delays (figure $1 \mathrm{~b}$ ).

*taravat@physik.tu-berlin.de; phone +49 3031425565

Photons Plus Ultrasound: Imaging and Sensing 2018, edited by Alexander A. Oraevsky, Lihong V. Wang, Proc. of SPIE Vol. 10494, 1049431 · C 2018 SPIE · CCC code: 1605-7422/18/\$18 · doi: 10.1117/12.2290223 
a)

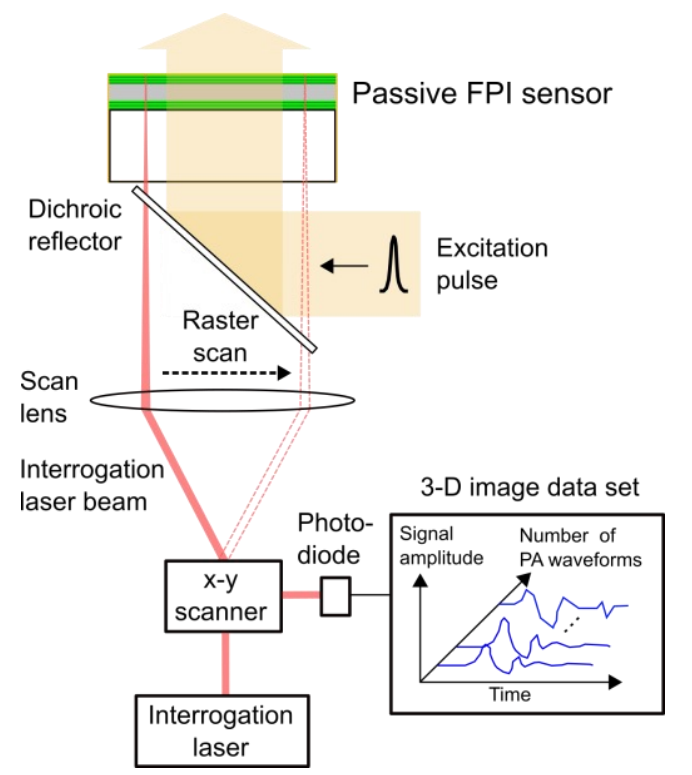

b)

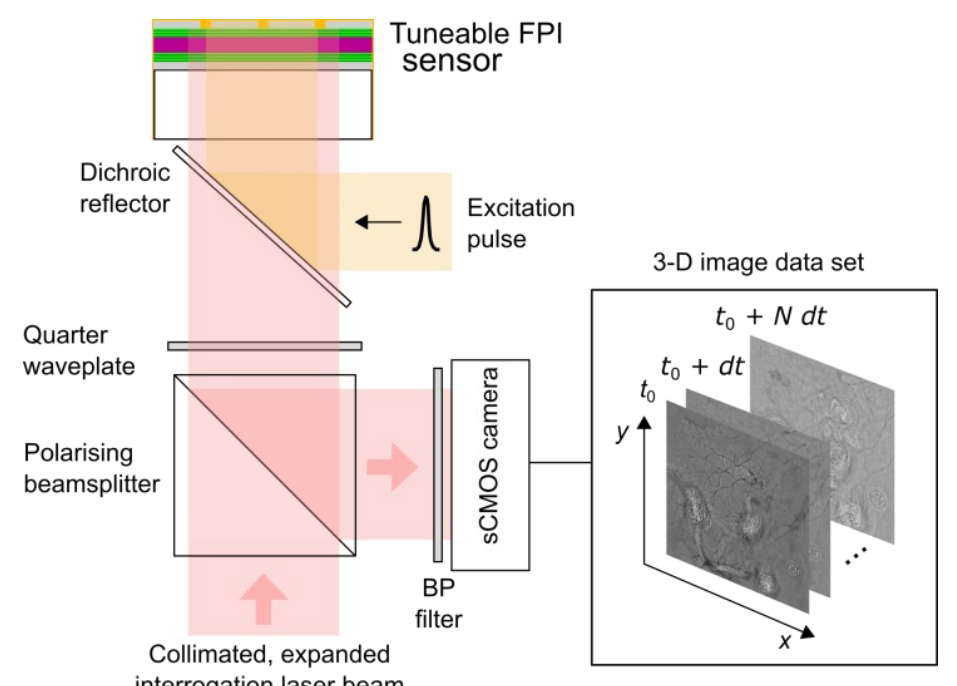

Figure 1. a) Raster scanning interrogation scheme, b) Proposed parallelised interrogation scheme for PA imaging system.

\section{MATERIALS AND METHODS}

\subsection{Fabry-Perot polymer film sensor fabrication}

In this study, a passive FPI ultrasound sensor consisting of dielectric mirrors made of $\mathrm{Ta}_{2} \mathrm{O}_{5}$ and $\mathrm{SiO}_{2}$ and a $20 \mu \mathrm{m}$ Parylene spacer deposited onto a COP substrate was used. The transmission spectrum of dielectric mirrors is shown in figure 2. The mirrors were designed to be highly transparent in the near infra-red (NIR) window $(600-1000 \mathrm{~nm})$ to allow backward-mode operation [1], and highly reflective around $520 \mathrm{~nm}$ in order to match the spectral sensitivity (quantum efficiency) of the camera.

To calculate the frequency response of the designed FPI sensor, an experimentally validated 1-D numerical model of the sensor [7] was implemented in $\mathrm{k}$-Wave [8]. The acoustic impedances and layer thicknesses of the materials used in the fabrication of FPI sensor were used as model input parameters. The acoustic source was modelled as a Gaussian distribution $(\mathrm{FWHM}=5 \mu \mathrm{m}$ ) and located adjacent to the sensor to ensure a broadband acoustic frequency spectrum similar to that observed in PA waves. 


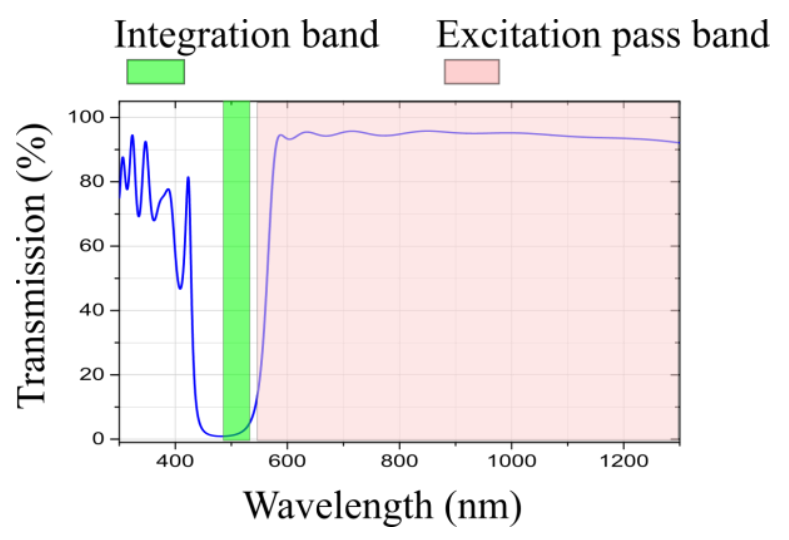

Figure 2. Transmission spectrum of FPI dielectric mirrors.

\subsection{Experimental setup}

A schematic of camera-based system for parallelised PA signal detection is shown in figure 3a. The acoustic transients incident on the FPI sensor are detected by mapping the temporal variation of the reflected optical field provided by an interrogation laser using a sCMOS camera. The measurement of the acoustically modulated component was mapped in $2 \mathrm{D}$ using the camera by capturing images at increasing $d t$. A single image would therefore represent the 2D distribution of the optical phase difference at a specific time point. To acquire a 3D PA image data set, multiple 2D images were recorded at different time delays $(N$ - number of measured images, $d t$-time increment) with respect to the excitation pulse $\left(t_{0}\right)$. An illustration of the signal detection is depicted in figure $3 \mathrm{~b}$. The reflected field was also measured using a Si photodiode for comparison. Details of the instruments of the system, optical interrogation system and parallelised PA signal acquisition scheme are provided below.

a)

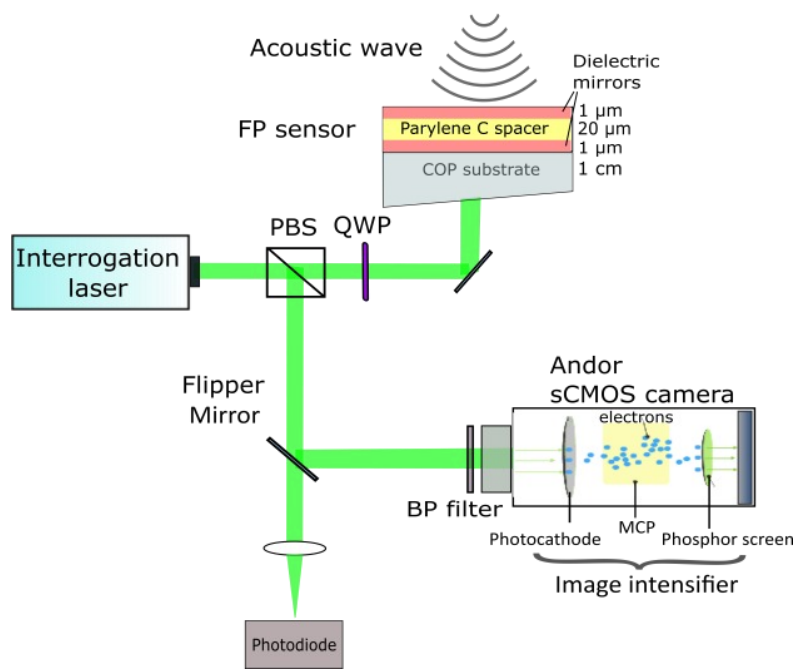

b)

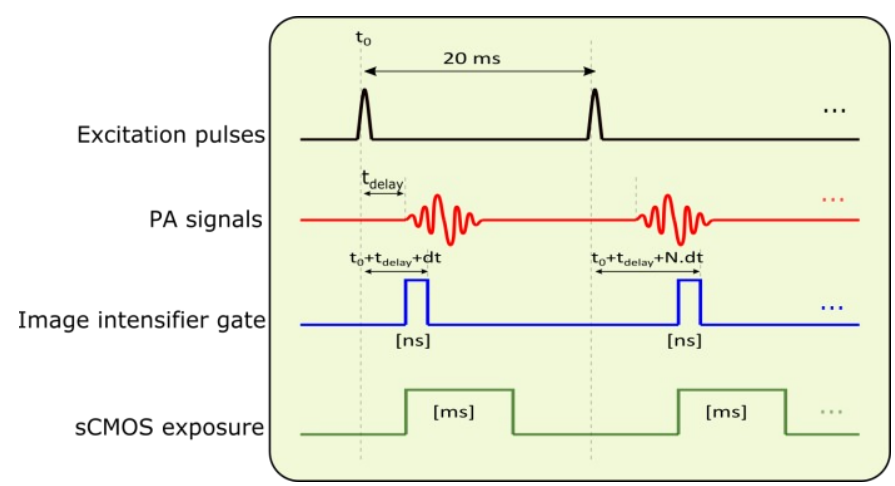

Figure 3. a) Camera-based system for parallelised PA signal detection, b) Sequence diagram of PA signal acquisition. 


\subsubsection{Comparison of ultrasound signals acquired using the photodiode and the SCMOS camera}

To measure the signal to noise ratio (SNR) of the camera-based system, a PZT ultrasound (US) transducer with $7 \mathrm{~mm}$ diameter active area and $2.25 \mathrm{MHz}$ centre frequency (SONOTEC, Germany) was used. The transducer was connected to a waveform generator (Agilent 33522A, USA), which provided sine wave bursts with 8 periods at a frequency of 2.25 $\mathrm{MHz}$, matching the centre frequency of the ultrasound transducer (figure 4a). US signals were generated in a water bath at a distance of $14 \mathrm{~mm}$ from the sensor. An FPI sensor with $20 \mu \mathrm{m}$ polymer spacer thickness was interrogated by a focused beam of a $532 \mathrm{~nm} \mathrm{cw}$ laser (WITec, Germany) at a single point at the center of the pressure field. The beam reflected from the sensor was directed via a polarizing beam splitter onto a Si photodiode-transimpedance amplifier configuration (Hamamatsu S5973) with dc- and ac-coupled outputs. The dc- and ac-coupled outputs were connected to a $350 \mathrm{MHz}$ digitizing oscilloscope (DSO) with a dynamic range of 12-bit, which was used to record the time-varying reflected optical power modulation produced by the incident acoustic wave.

In a second experiment, the optical field reflected from the FPI sensor was directed to an iStar sCMOS camera (Andor) using a flip mirror (Thorlabs). The camera includes an image intensifier providing a minimum optical gate width of 2.4 ns. The light is detected by a CMOS chip with a maximum image array size of $2560 \times 2160$ pixels with $6.5 \mu \mathrm{m}^{2}$ pixel size and 50 frames/second (full frames) acquisition times. For the measurement reported here, an optical gate width of $20 \mathrm{~ns}$ and a region of interest (ROI) of $100 \times 100$ pixels were used.

\subsubsection{Parallelised PA signal acquisition system}

To generate PA signals, an excitation laser was used (Innolas Spitlight 1000) which consists of a Nd:YAG pump laser and a type II optical parametric oscillator (OPO) with pulse repetition rate (PRF) of $30 \mathrm{~Hz}$. The laser produced $6 \mathrm{~ns}$ pulses which were coupled into a $1.5 \mathrm{~mm}$ multimode fibre to homogenise the beam. The fluence at the target was 8 $\mathrm{mJ} / \mathrm{cm}^{2}$. Broadband PA signals were generated in an India ink solution with an absorption coefficient $\left(\mu_{\mathrm{a}}\right)$ of $9 \mathrm{~mm}^{-1}$ (figure 4b). The FPI sensor was interrogated using a collimated beam of $2 \mathrm{~mm}$ diameter. The beam was measured over an ROI of $100 \times 90$ pixels $\left(3.7 \mathrm{~mm}^{2}\right)$ of the camera. The corresponding PA signals are shown in figure 7 . Two data sets were acquired using an optical gate width of $10 \mathrm{~ns}$ and $40 \mathrm{~ns}$, $d t$ of $10 \mathrm{~ns}$ (equal to $100 \mathrm{MHz}$ sampling frequency) and 40 $\mathrm{ns}$ (equal to $25 \mathrm{MHz}$ sampling frequency) and micro-channel plate gains of 300 and 100, respectively.

a)

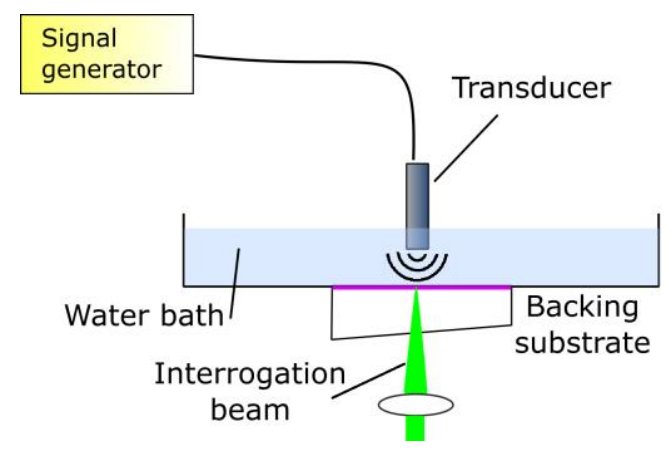

b)

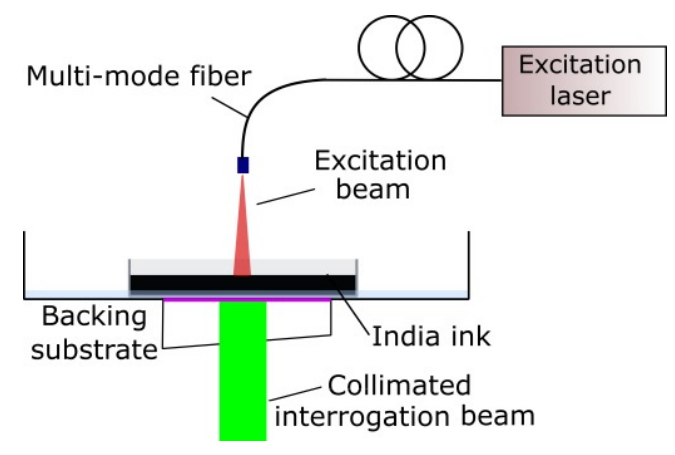

Figure 4. a) Setup for measurement of SNR, b) Setup for parallelised detection of PA signals generated in India ink. 


\section{RESULTS}

Frequency response of the FPI sensor with hard dielectric mirrors modified for $520 \mathrm{~nm}$ is depicted in figure 5 and shows a broadband acoustic response of $100 \mathrm{MHz}$.

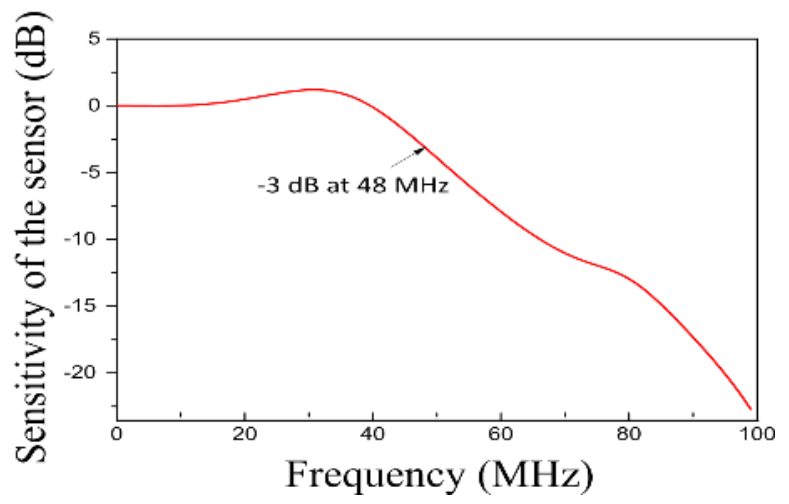

Figure 5. Modelled acoustic frequency response of the FPI sensor.

Figure 6 shows the signal to noise ratio of ultrasound signals measured by the photodiode- and sCMOS camera- based setups, $\mathrm{SNR}=8.5$ and $\mathrm{SNR}=7.5$, respectively. The comparison shows that both systems generally deliver comparable acoustic sensitivity. Due to low dynamic range of the oscilloscope (DSO) a digitization error can be seen in the recorded dc measurement (figure 6a).

The results of the parallelised PA signal detection using a sCMOS camera are shown in figure 7. 400 images were acquired by the camera in $10 \mathrm{~ns}$ and $40 \mathrm{~ns}$ steps. The time-course of the reflected optical power was detected at three different locations, each represents an active element within the acoustically sensitive region of the FPI sensor. It should be noted that due to the inhomogeneity of the optical thickness of the passive FPI used here not all image pixels represented acoustically sensitive elements. SNR calculations of PA signals of ROI 3 were made as an example, resulting 5.22 and 13.21 for $10 \mathrm{~ns}$ and $40 \mathrm{~ns}$ optical gate widths, respectively. This yields an approximately $\sqrt{ } 4$ times stronger signal at $40 \mathrm{~ns}$ gate width according to accumulating more photon flux.

a)

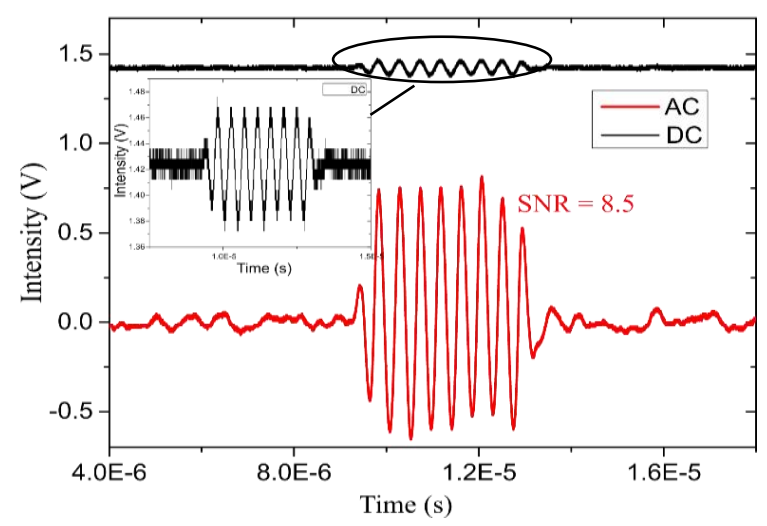

b)

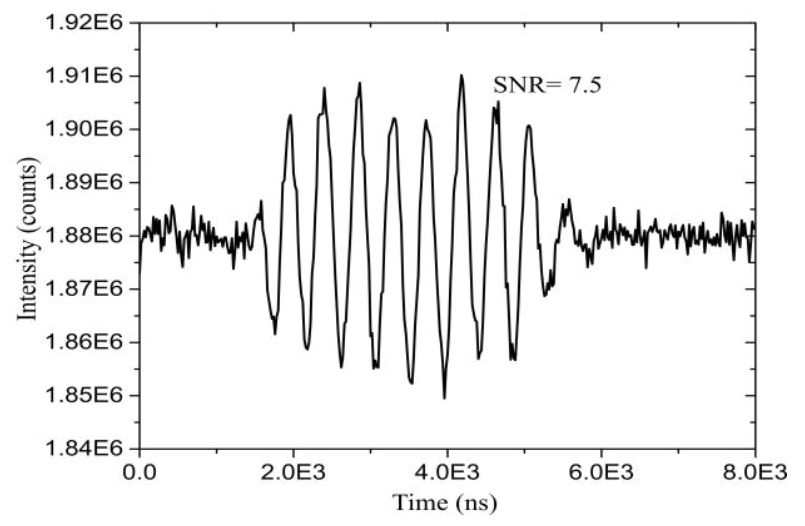

Figure 6. Detected US signals by a) Photodiode ac (red) and dc (black) outputs, b) sCMOS camera. 
a)

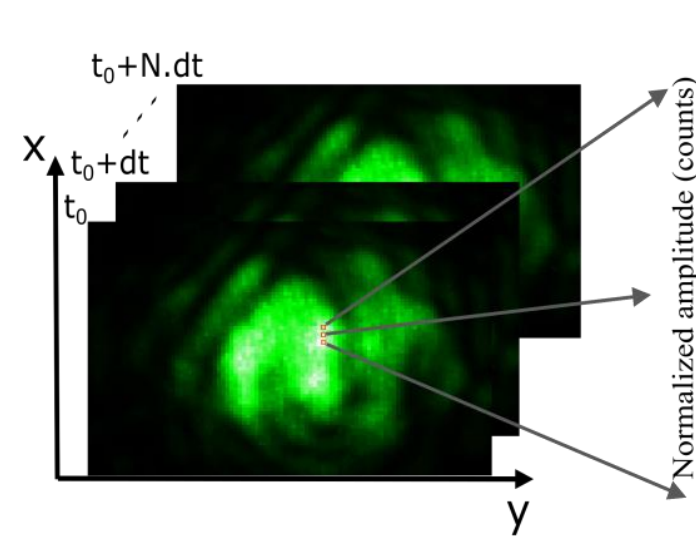

b)

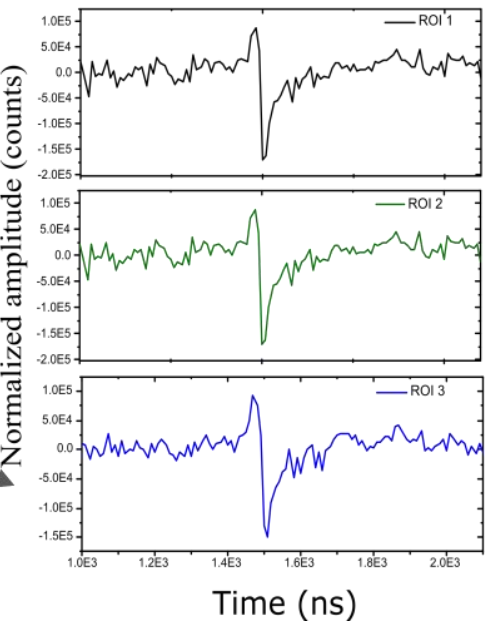

c)

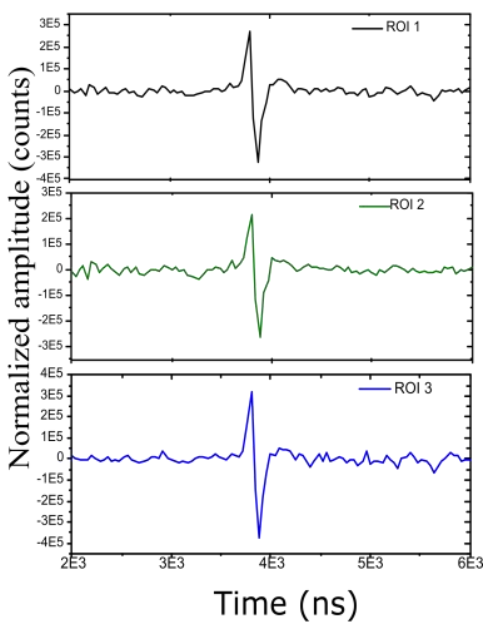

Figure 7. Parallelised PA signal detection. a) Image series acquired by sCMOS camera at increasing time courses, b) PA signal acquired with optical gate width of $10 \mathrm{~ns}$ c) PA signal acquired with optical gate width of $40 \mathrm{~ns}$.

\section{DISCUSSIONS AND CONCLUSION}

A parallelised PA signal detection using a camera-based interrogation scheme was developed. This new system has the potential to reduce image acquisition times by orders of magnitude compared to the single-beam raster scanning method. Comparison of the SNR of the raster scanning system and parallelised interrogation system suggests that the camerabased system has the potential to provide similar acoustic sensitivity.

To illustrate the potential improvement in image acquisition time, the parallelised and raster-scan approaches are compared. Assuming the total PA signal duration to be $10 \mu \mathrm{s}$ and an image sampling rate of one image every $40 \mathrm{~ns}$, i.e. a total of 250 images, at a PRF of $50 \mathrm{~Hz}$ of excitation laser (similar to the frame rate of the sCMOS chip), then the acquisition time using parallelised interrogation scheme is approximately 5 seconds. This would yield for around 90000 waveforms (i.e. number of image pixels). By comparison, the acquisition times needed for the same number of waveforms with single beam raster scanning is about 30 minutes.

\section{REFERENCES}

[1] Zhang E., Laufer J., \& Beard P., "Backward-mode multiwavelength photoacoustic scanner using a planar Fabry-Perot polymer film ultrasound sensor for high-resolution three-dimensional imaging of biological tissues", Appl. Opt. 47, 561- 577 (2008)

[2] Laufer J., Johnson P., Zhang E., Treeby B., Cox B., Pedley B., Beard P., "In vivo preclinical photoacoustic imaging of tumor vasculature development and therapy", Journal of Biomedical Optics 17(5), 056016 (2012)

[3] Cox B., Laufer J.G., Arridge S.R., Beard P., "Quantitative spectroscopic photoacoustic imaging: a review", Journal of Biomedical Optics 17(6), 061202 (2012)

[4] Laufer J., Norris F., Cleary J., Zhang E., Treeby B., Cox B., Johnson P., Scambler P., Lythgoe M., Beard P., "In vivo photoacoustic imaging of mouse embryos", Journal of Biomedical Optics 17(6), 061220 (2012)

[5] Märk J. et al., "Dual-wavelength 3D photoacoustic imaging of mammalian cells using a photoswitchable phytochrome reporter protein" Commun. Phys. (2018). <in press.>

[6] Huynh N., Ogunlade O., Zhang E., Cox B., and Beard P. "Photoacoustic imaging using an 8-beam Fabry-Perot scanner", Proc. of SPIE, Vol. 9708, paper 97082L (2016)

[7] Buchmann J., Zhang E., Scharfenorth C., Spannekrebs B., Villringer C., Laufer J., "Characterization and modeling of Fabry-Perot ultrasound sensors with hard dielectric mirrors for photoacoustic imaging" Applied Optics 56, 5039-5046 (2017)

[8] Treeby B., Cox B., "k-Wave: MATLAB toolbox for the simulation and reconstruction of photoacoustic wave fields", Biomed. Opt., vol. 15, no. 2, p. 021314 (2010) 